\title{
Quantum Chemistry, Anomalous Dimensions, and the Breakdown of Fermi Liquid Theory in Strongly Correlated Systems
}

\author{
Gabriel Kotliar and Qimiao Si \\ Serin Physics Laboratory, Rutgers University \\ Piscataway NJ 08854 USA
}

\begin{abstract}
We formulate a local picture of strongly correlated systems as a Feynman sum over atomic configurations. The hopping amplitudes between these atomic configurations are identified as the renormalization group charges, which describe the local physics at different energy scales. For a metallic system away from half-filling, the fixed point local Hamiltonian is a generalized Anderson impurity model in the mixed valence regime. There are three types of fixed points: a coherent Fermi liquid (FL) and two classes of self-similar (scale invariant) phases which we denote incoherent metallic states (IMS). When the transitions between the atomic configurations proceed coherently at low energies, the system is a Fermi liquid. Incoherent transitions between the low energy atomic configurations characterize the incoherent metallic states. The initial conditions for the renormalization group flow are determined by the physics at rather high energy scales. This is the domain of local quantum chemistry. We use simple quantum chemistry estimates to specify the basin of attraction of the IMS fixed points.
\end{abstract}

To appear in the Proceedings of the European Physical Society Meeting, Regensburg, Germany, March 1993

\section{A. Introduction}

The anomalous normal state properties in the high $T_{c}$ superconductors have led to current theoretical interests in addressing how electron-electron interactions can result in non-Fermi liquid fixed points. [1.2] For interacting fermion systems in one dimension, the breakdown of Fermi liquid theory occurs for infinitesimal values of the interaction and can therefore be studied using a renormalization group (RG) which is perturbative in the interaction strength. [3]

In more than one dimension, such a perturbative RG analysis has led to the conclusion that, Fermi liquid theory describes weakly interacting systems with regular dispersion. [4] Therefore, the breakdown of Fermi liquid theory, if it occurs, is necessarily non-perturbative in the interaction strength. Most of the previous approaches to this problem, however, start with quasiparticle states and ask how singularities can occur in the interaction vertex between these quasiparticles. Motivated both by the non-perturbative character of the problem and by the local character of the interactions we approach the problem of the breakdown of Fermi Liquid theory from the opposite end: the expansion around the atomic limit suggested in Hubbard's early work. [5] The main difficulties with this approach is that a direct perturbation expansion around the atomic limit is a classic example of a singular perturbation problem which 
requires a zeroth order solution before the perturbation expansion can be attempted. This zeroth order solution is not available except at very high temperatures. For these reasons, this approach was largely abandoned by most of the condensed matter community. However, recent non perturbative solutions of lattice models in large dimensions have revealed that in certain regime of parameters the Hubbard description becomes asymptotically valid at low energies. [8.9] An important technical advance was the extension of early work of Haldane on the Anderson model, [10] through which we have developed a systematic renormalization group method for arbitrary set of local configurations. This allows us to control the perturbation expansion around the atomic limit and, in the regime where non-trivial phases occur, calculate with some rigor the low energy properties of incoherent metallic states (IMS). [9] We stress that at the end one ought to be able to express our results, derived using a local method, in a language that would make contact with perturbative calculations in the interactions. When this is done we may be able to identify some similarities between our ideas and those of the theories cited earlier. In particular, we stress that the phases we identify exhibit charge spin separation and power law type correlation functions. These properties have striking analogies to the Luttinger liquid in one dimension, [3] and to the uniform RVB in the gauge theory formulation. [11]

In this paper we outline a general framework for determining the conditions of the breakdown of Fermi liquid theory, from a local analysis. We explain the physical meaning of the parameters which control the local aspects of this phenomena, i.e. the RG charges, as well as the reasons why they are scale dependent. In an incoherent metallic state the evolution of the RG charges as a function of length scale, can be determined analytically at sufficiently low energies. At high energies the initial conditions of the flows can be determined by simple perturbative quantum chemistry calculations. Assuming that the two regimes can be matched smoothly we specify the explicit parameter range for which a two band model falls in the basin of attraction of the incoherent metallic phase.

\section{B. The Local View of a Metallic System}

The local description of a strongly correlated metal starts from selecting a relevant set of "atomic configurations" in a cluster of atoms. The simplest realization of this idea would take a cluster consisting of a single site with a small set of atomic configurations. However, more general choices (larger clusters or more general molecular configurations) are possible. Locally the dynamics within this Hilbert space is described by carrying out a Feynman sum over all the local histories. Each history is described by a succession of hops between the atomic configurations. The weight of each path depends on the local physics and the coupling to the neighboring sites.

This idea can be made precise, in any number of dimensions, by following Georges and Kotliar [7] in writing down a path integral representation of the partition function and integrating out all the electrons except for those in the selected cluster. The result of this process is an effective action which describes the influence of the environment on the quantum tunneling between the configurations (i.e. the "atomic states") of the cluster. Formally, [7]

$$
\mathrm{e}^{-\mathrm{S}_{\text {eff }}(\Gamma)}=\int \Pi^{\prime} d \psi^{+} d \psi \mathrm{e}^{-\mathrm{S}\left[\psi^{+}, \psi\right]}
$$

where $\Pi^{\prime}$ involves all sites except the one we are interested, and

$$
\mathrm{S}_{\mathrm{eff}}(\Gamma)=\sum_{n=2}^{\infty} \int d \tau_{i} d s_{j} \Gamma_{\alpha_{1} \ldots \alpha_{n} \beta_{1} \ldots \beta_{n}}^{\tau_{1} \ldots \tau_{n} s_{1} \ldots s_{n}} \psi_{\alpha_{1}}^{+}\left(\tau_{1}\right) \ldots \psi_{\alpha_{n}}^{+}\left(\tau_{n}\right) \psi_{\beta_{1}}\left(s_{1}\right) \ldots \psi_{\beta_{n}}\left(s_{n}\right)
$$


where $\psi_{\alpha}$ are orbitals of interest.

The exact determination of the various $\Gamma_{\alpha}$ for models in finite spatial dimensions is a difficult problem. In infinite dimensions, [7] the effective action simplifies and contains only a quadratic term, and quartic terms which are given by the bare local interaction. In the absence of broken spatial symmetries, the coefficient of the quadratic term, which in previous publications was denoted by $-G_{0}{ }^{-1}$, can be simply related to the local correlators calculated with this action. This paves the way for exact solutions of correlated models in large dimensionality. We stress, however, that at this point our general framework is not restricted to large dimensions. What the large dimensionality limit allows us to do is to calculate explicitly the effective actions and the local correlation functions. In addition, from the knowledge of the local physics one can reconstruct all the lattice correlation functions. [6, /7]

This local view opens the road to a classification of all the possible low energy behaviors of metallic systems in the limit of infinite dimensions. In finite dimensions this local analysis can give a qualitative understanding of the nature of the metallic state. The key point is that, while generically the problem associated with $S_{\text {eff }}$ involves many configurations, and hence is not universal and belongs to the realm of quantum chemistry, at low energies the physical content of $S_{\text {eff }}$ simplifies since it has to describe the fluctuations between a very small number of low energy configurations. If the configurations have the same charge, we are in an insulating situation. The typical example for such a case is provided by the Mott insulating phase which has been studied recently in infinite dimensions. [8] If the system is metallic, it has to fluctuate between low energy configurations having different charges. A natural possibility is that, the low energy configurations with different charges are a spin singlet and a spin doublet. If the $S_{\text {eff }}$ describes a system interacting with a bath with a non singular density of states, one can write down the most general model which contains a singlet and a doublet interacting and hybridizing with a bath: this is the generalized Anderson impurity model in the mixed valence regime.

From the local point of view, the survival or the demise of Fermi liquid theory depends on how the quantum mechanical hopping amplitudes are renormalized as we go to lower energies. If these quantum mechanical hopping amplitudes are renormalized to infinity, coherent hopping results in at long time scales, and the system is a Fermi liquid. If they are renormalized to zero, at long time scales the transitions between the local states occur incoherently, and the resulting state is an incoherent metallic state (IMS). That a transition between incoherent and coherent tunneling does occur in two level systems coupled to an environment, is well understood in the context of the macroscopic quantum tunneling. 12] To extend this idea to the strong correlation problem, we identify the coordinate that performs the quantum tunneling with the atomic configurations of the correlated system. We then carry out an RG analysis describing the renormalization of the tunneling amplitudes as a function of length scale.

The minimal model in this case is a three level system, with a singlet state and a spin doublet. The fact that the coordinate lies in a three dimensional space brings together the physics of spin and charge fluctuations, and gives rise to a novel zero temperature quantum phase transition, the mixed valence critical point, which is in a new universality class [9]. A detailed analysis of this critical point can be found in our previous publications. Here we discuss the physical content of our results, and stress the generality of our ideas.

From the physical point of view, our picture has three degrees of freedom which fluctuate without an energy barrier. The spin flips are generically massless, since spin up and spin down have the same energy due to the global spin rotation 
invariance. The physical reason why the transition between states with different local charges is massless is more subtle, since it is not protected by any symmetry. In fact the breaking of local U(1) gauge invariance is characteristic of metallic state. The essential point [13] is that in order for an incoherent state to be metallic, it is necessary to allow for charge transfer between the localized degrees of freedom and the bath. This can only happen if the local degree of freedom is in equilibrium with the conduction electron bath. This requires the heavy level to be at the chemical potential causing the empty and the occupied states to have the same energy.

This is reminiscent of recent approaches based on the slave boson technique [1]. The slave boson describes the local charge fluctuation and remains incoherent in the uncondensed phase. In both treatments we see that, in the IMS, the local gauge invariance which is broken by the coherent hopping is restored at low energies.

We therefore find a unusual situation, that the impurity model that the large d system maps onto has a larger symmetry than one would have naively expected. The metallic strongly correlated state is locally described by an impurity model, a generalized Anderson model, in the mixed valence regime. [7,9]

\section{The Universal Low Energy Behavior}

Fixed Point Hamiltonian The generalized Anderson impurity Hamiltonian describes three local states coupled to an electron bath with a smooth density of states:

$$
\begin{aligned}
H= & \sum_{k \sigma}\left(\epsilon_{k}-\mu\right) c_{k \sigma}^{+} c_{k \sigma}+E_{d}^{o} d_{\sigma}^{+} d_{\sigma}+\frac{U}{2} \sum_{\sigma \neq \sigma^{\prime}} d_{\sigma}^{+} d_{\sigma} d_{\sigma^{\prime}}^{+} d_{\sigma^{\prime}} \\
& +\sum_{\sigma} t\left(d_{\sigma}^{+} c_{\sigma}+h . c .\right)+V_{1} \sum_{\sigma, \sigma^{\prime}} d_{\sigma}^{+} d_{\sigma} c_{\sigma^{\prime}}^{+} c_{\sigma^{\prime}}+\frac{V_{2}}{4} \sum_{\sigma_{1}, \sigma_{2}, \sigma_{3}, \sigma_{4}} \tau_{\sigma_{1} \sigma_{2}} \cdot \tau_{\sigma_{3} \sigma_{4}} d_{\sigma_{1}}^{+} d_{\sigma_{2}} c_{\sigma_{3}}^{+} c_{\sigma_{4}}
\end{aligned}
$$

where $\tau$ label the Pauli matrices. The local spin singlet $\mid s>$ and spin doublet $\mid \sigma>$ are denoted by the vacuum $\mid 0>$ with $E_{0}=0$ and the singly occupied states $d_{\sigma}^{+} \mid 0>$ with $E_{\sigma}=E_{d}^{o}$ respectively. An infinite onsite interaction, $U=\infty$, is introduced to enforce the three dimensional restricted configuration space. The hybridization $t$, the density-density interaction $V_{1}$, and the spin exchange interaction $V_{2}$ describe the generic couplings between the local states and the electron bath.

Here $\epsilon_{k}$ describes the dispersion of the electrons in the bath. Such a dispersion is determined by solving the self consistency condition. We envision that below a scale $t_{p}$ (the low energy regime) the density of states is featureless and can be taken to be a constant. We have shown that such a non-singular density of states is self-consistent. [14]

At energy scales lower than $t_{p}$, we write the partition function and the local correlation functions as Feynman sums over trajectories in the local configuration space. We envision calculating $\mathrm{N}$ point correlation functions of Hubbard operators $X_{\alpha, \beta}$ at $\mathrm{N}$ values of imaginary time. The insertion of Hubbard operators force the system to be at certain configuration or to flip configurations at given values of imaginary time. The amplitude for this process is the sum over all trajectories consistent with these constraints. The weight for each trajectory depends of course on the reaction of the electrons in the neighboring sites to a given local trajectory. This exact representation is due to Haldane. [10] It was recently extended by us, to incorporate generic local configurations and couplings in all channels, using a simple bosonization procedure. It has the form: 


$$
\frac{Z}{Z_{0}}=\sum_{n=0}^{\infty} \sum_{\alpha_{1}, \ldots, \alpha_{n}} \exp \left(-S\left[\tau_{1}, \ldots \tau_{n}\right]\right)
$$

where

$$
\begin{aligned}
S\left[\tau_{1}, \ldots \tau_{n}\right]= & \sum_{i<j}\left(K\left(\alpha_{i}, \alpha_{j}\right)+K\left(\alpha_{i+1}, \alpha_{j+1}\right)-K\left(\alpha_{i}, \alpha_{j+1}\right)-K\left(\alpha_{i+1}, \alpha_{j}\right)\right) \ln \frac{\left(\tau_{j}-\tau_{i}\right)}{\xi_{o}} \\
& -\sum_{i} \ln \left(y_{\alpha_{i} \alpha_{i+1}}\right)+\sum_{i} h_{\alpha_{i+1}} \frac{\left(\tau_{i+1}-\tau_{i}\right)}{\xi_{o}}
\end{aligned}
$$

Here $\tau_{i}$, for $i=1, \ldots, n$, labels the hopping event from local state $\mid \alpha_{i}>$ to $\mid \alpha_{i+1}>$, and $\xi_{o}$ is the ultraviolet inverse energy cutoff.

The fugacities $y_{\alpha, \beta}$ describe the amplitudes for quantum hopping between the configurations $\mid \alpha>$ and $\mid \beta>$. The charge fugacity describes the hopping between two local states with different charges and is determined by the hybridization term, $y_{0, \sigma}=y_{t}=t \xi$. Similarly, the spin fugacity describes the hopping between two local states with different spin quantum numbers and is determined by the transverse component of the exchange coupling, $y_{\sigma \neq \sigma^{\prime}}=y_{j}=\frac{V_{2}^{\perp}}{2} \xi$. The fields $h_{\alpha}$ describe the energy splitting among the local configurations. They have to be introduced since generically there is no symmetry between the local many body configurations. In our three state problem, there exists a spin rotational symmetry but not a symmetry between spin and charge. Therefore, we introduce a field related to the $d$ level $E_{d}$, i.e. $h_{0}=-\frac{2}{3} E_{d} \xi$ and $h_{\sigma}=\frac{1}{3} E_{d} \xi$.

The logarithmic interaction between the hopping events (which can be thought of as defects) describe the reaction of the electron bath towards disturbances at the impurity sites. The bare values of the stiffness constants, $\epsilon_{t}=-K(0, \sigma)$ and $\epsilon_{j}=-K\left(\sigma, \sigma^{\prime} \neq \sigma\right)$ are determined by the interaction strengths. They provide the initial conditions of the renormalization group flow, and will be discussed in detail in the next section. The logarithmic interaction in the action (5) can be written as a classical "spin" model with long range $\frac{1}{\tau^{2}}$ interaction, $\sum_{i<j} K\left(\alpha_{i}, \alpha_{j}\right)\left(\frac{\xi_{0}}{\tau_{i}-\tau_{j}}\right)^{2}$. 15] This allows us to adapt Cardy's RG equations [15] to our problem.

Scaling Equations The renormalization group equations describe how the fugacities evolve as a function of length scale. A detailed derivation can be found in Refs. [9]. Here we quote the results for the spin $\frac{1}{2}$ case,

$$
\begin{aligned}
& d y_{t} / d \ln \xi=\left(1-\epsilon_{t}\right) y_{t}+y_{t} y_{j} \\
& d y_{j} / d \ln \xi=\left(1-\epsilon_{j}\right) y_{j}+y_{t}^{2} \\
& d \epsilon_{t} / d \ln \xi=-6 \epsilon_{t} y_{t}^{2}+\epsilon_{j}\left(y_{t}^{2}-y_{j}^{2}\right) \\
& d \epsilon_{j} / d \ln \xi=-2 \epsilon_{j}\left(y_{t}^{2}+2 y_{j}^{2}\right) \\
& d E_{d} \xi / d \ln \xi=\left(y_{t}^{2}-y_{j}^{2}\right)+E_{d} \xi\left(1-3 y_{t}^{2}\right)
\end{aligned}
$$

In the renormalization of the fugacities, the linear terms give the associated anomalous dimensions, while the quadratic terms reflect the non-abelian nature of our three state problem. The renormalization of the stiffness constants reflect the correction to interactions induced by the fugacities. Finally, in the renormalization of the energy level, the $y_{t}^{2}$ and $y_{j}^{2}$ terms arise due to the particle-hole asymmetry. [10]

Universality Classes The defects are bound together when the attraction among them are strong enough. This leads to fugacities which are renormalized to zero. As in the Kosterlitz-Thouless transition in the XY magnet, 
unbinding of the defects occurs when the attraction becomes weaker. In our case, $\epsilon_{t}$ and $\epsilon_{j}$ reflect the strength of the long range interactions between the defects.

Since the spin and charge defects are coupled, the unbinding of these defects can occur at the same time, or at different stages. We find that, there can be three kinds of fixed points.

The strong coupling mixed valence fixed point occurs when both the spin and charge defects are unbound. Here both the hybridization and the Kondo exchange are relevant, and therefore both the local spin and local charge degrees of freedom are quenched. This state is in the same universality class as the usual strong coupling phase of the Anderson model. The basin of attraction of this case is given by $\epsilon_{t}<1$ and a range of $\epsilon_{j}<1$ when $\epsilon_{t}>1$.

The weak coupling mixed valence fixed points occur when both the spin and charge defects are bound. Here both the hybridization and the Kondo exchange are irrelevant, and therefore neither the local spin nor the local charge degrees of freedom are quenched. The basin of attraction for this case is determined by $\epsilon_{t}>1$ and $\epsilon_{j}>1$.

The intermediate coupling mixed valence fixed points occur when the spin defects are unbound while the charge defects are bound. [16] The hybridization is irrelevant, while the Kondo exchange is relevant. Therefore, local spins are quenched while local charges are not. The basin of attraction of this case is in a range within $\epsilon_{t}>1$ and $\epsilon_{j}<1$. Such an intermediate phase is the analog of the hexatic phase in the two dimensional melting problem, in which unbinding occurs for dislocations but not for disclinations. [17]

As the chemical potential is varied, the strong coupling mixed valence state occurs within a crossover region over a range of chemical potential. This range is determined by the renormalized hybridization, as was characterized by Haldane. [10] For the weak coupling and intermediate coupling cases, the renormalized hybridization is zero. Therefore, the mixed valence states occur at a critical chemical potential $\mu_{c}$. As $\mu$ deviates from $\mu_{c}$, the mixed valence states eventually crossover into either an empty orbital or a local moment regime. It should be emphasized that, the pinning effect discussed previously led to the conclusion that, $\mu_{c}$ corresponds to a range of total electron densities.

Within the strong coupling mixed valence state, an energy scale (renormalized Fermi energy) is generated below which quasiparticles appear. The low energy physics is described by a coherent Fermi liquid.

In the weak coupling mixed valence state, no such energy scale is generated, and the system is scale invariant. The renormalized Fermi energy vanishes, resulting in a non-Fermi liquid state-an incoherent metallic state. Local correlation functions, both in spin and charge channel, show algebraic behavior. Spin and charge excitations are asymptotically decoupled.

In the intermediate coupling mixed valence state, a coherent energy scale is generated in the spin channel, but not in the charge channel. We have therefore a spin-charge separated state with coherent spin excitations and incoherent charge excitations. This state resembles the spin-charge separated state that occurs in the gauge approach when slave boson is not condensed, 11 which has coherent 'spinon' excitations and incoherent 'holon' excitations.

Critical Behavior The description of the physics in terms of defects allows us to make several general statements about the zero temperature quantum phase transitions between the various states we defined. These are binding unbiding transitions in $0+1$ dimensions.

The transitions are characterized by the collapse of an energy scale,

$$
\epsilon_{F} \sim \epsilon_{0} \mathrm{e}^{-\frac{1}{\left(\epsilon-\epsilon_{c}\right)^{\eta}}}
$$


For the transition between the Fermi liquid and the weak coupling non-Fermi liquid states, the binding-unbinding transition for spin defects is driven by that of the charge defects. Therefore, $\epsilon_{F}$ corresponds to the Fermi energy of the Fermi liquid. For the two stage transition from the Fermi liquid, through the intermediate coupling non-Fermi liquid state, to the weak coupling non-Fermi liquid state, the $\epsilon_{F}$ corresponds to the coherence energies in the spin channel and in the charge channel respectively. The exponent $\eta=\frac{1}{2}$ for the transitions from the weak coupling phase to the strong coupling phase and from the intermediate coupling phase to the strong coupling phase, while $\eta=1$ for the transition from the weak coupling phase to the intermediate coupling phase due to rotational invariance.

\section{The Non-Universal High Energy Behavior-Quantum Chemistry}

The low energy behavior of a metallic system is universal in the sense that the behavior of the local correlation functions is controlled by the different fixed points of the impurity hamiltonian described in the previous section.

In a previous publication we arrived at that impurity model starting from an extended two band Hubbard model on a lattice in the limit of infinite dimensions [9]. We stress however that the low energy description of the previous section is in fact much more general. There are many lattice hamiltonians, whose local physics at low energies is described by the renormalization group equations (6). In particular one can arrive at low energies at the impurity model of the previous section starting from a metallic one band system. The role of the "light electrons" of Ref. [9] is now played by the incoherent part of the one particle Green's function of the one band system.

In fact one can arrive at our local low energy description for a correlated metallic state from very general considerations. The local configurations are expected to have different spin and charge quantum numbers, and can be described by a spin doublet and a spin singlet. The electron bath should have non-zero density of states near the Fermi level. They are coupled generically in both spin and charge sectors. Finally we also expect a one to one correspondence between the local degrees of freedom and the degrees of freedom of the bath, as embodied in the self consistency condition.

While these assumptions are simple and natural they are by no means exclusive. Ruckenstein, Varma and collaborators have suggested that an impurity model with a bath with several channels of screening electrons is related to the copper oxides. 18] These impurity models have not been derived from a lattice model in large dimensions.

We now turn to the crucial question of the determination of the initial conditions for our RG flows. This is a non universal high energy question that depends on system to system.

In an extended Hubbard model with the Lorentzian case, the width of the scaling regime $t_{p}$ equals the bare conduction electron bandwidth. The initial conditions are determined by the phases shifts of the conduction electron bath, which are directly related to the physical interaction strength. They are given as follows,

$$
\begin{aligned}
\epsilon_{t} & =\frac{1}{2}\left[\left(1-\frac{\delta_{2}}{\pi}-\frac{\delta_{1}}{\pi}\right)^{2}+\left(\frac{\delta_{1}}{\pi}-\frac{\delta_{2}}{\pi}\right)^{2}\right] \\
\epsilon_{j} & =\left(1-2 \frac{\delta_{2}}{\pi}\right)^{2}
\end{aligned}
$$

where the phase shifts are $\delta_{1}=\tan ^{-1}\left(\pi \rho_{o} V_{1}\right)$ and $\delta_{2}=\tan ^{-1}\left(\pi \rho_{o} V_{2} / 4\right)$, with $\rho_{o}$ the conduction electron density of states at the Fermi level. 
The basin of attraction for different phases given in the previous section can now be specified in terms of the phase shifts. The weak coupling non-Fermi liquid state occurs $\delta_{1} / \pi<-(\sqrt{3}-1) / 2$ and $\delta_{2}<0$. The intermediate coupling non-Fermi liquid state occurs over a range within $\delta_{1} / \pi<-(\sqrt{3}-1) / 2$ and $\delta_{2}>0$. Otherwise, a Fermi liquid state emerges at low energies.

To determine the basin of attraction of the various phases in more realistic models, a more elaborate treatment of the high energy region is necessary. One has to solve first the self consistency equation for the parameters of the impurity model, and then integrate out the high energy states of the impurity to arrive at the low energy effective impurity model. Previous work on the Hubbard model demonstrated that, the impurity models that describe the local physics of lattice problems are characterized by atomic like features (Hubbard bands) and narrow resonances near the Fermi level. To treat the atomic like features in a narrow band situation we can carry out a poor man's version of a quantum chemistry calculation to determine the initial conditions for the couplings that enter the low energy renormalization group flow.

To illustrate our idea, we parametrize the associated impurity problem, i.e. the $\Gamma_{\alpha}$ in $S_{\text {eff }}$ using the following Hamiltonian:

$$
\begin{aligned}
\mathrm{H}_{\mathrm{imp}}= & \epsilon_{d} d_{\sigma}^{+} d_{\sigma}+\frac{U}{2} \sum_{\sigma \neq \sigma^{\prime}} d_{\sigma}^{+} d_{\sigma} d_{\sigma^{\prime}}^{+} d_{\sigma^{\prime}}+\epsilon_{p} p_{o, \sigma}^{+} p_{o, \sigma}+\sum_{<i, j>} t_{i j}^{p}\left(p_{i \sigma}^{+} p_{j \sigma}+\text { h.c. }\right) \\
& -t\left(d_{\sigma}^{+} p_{o \sigma}+\text { h.c. }\right)+V_{1}^{o} \sum_{\sigma, \sigma^{\prime}} d_{\sigma}^{+} d_{\sigma^{\prime}} p_{\sigma^{\prime}}^{+} p_{\sigma^{\prime}}+V_{2}^{o} \sum_{\sigma, \sigma^{\prime}} d_{\sigma}^{+} d_{\sigma^{\prime}} p_{\sigma^{\prime}}^{+} p_{\sigma}
\end{aligned}
$$

Here $\epsilon_{d}$ and $\epsilon_{p}$ label the levels of the local $d$ and $p$ electrons, both of which are determined by the corresponding levels and the chemical potential of the lattice model. The Hubbard interaction $U$ is taken as infinite. $t_{01}^{p}$ reflects the coupling between $p$ electrons at the local and the nearest neighbor site. $t_{i j}^{p}$ parametrize the generic spectral function of the electron bath. We have also included the generic interaction parameters between the $d$ and $p$ electrons, a repulsive interaction $V_{1}^{o}$ in the density-density channel, and an antiferromagnetic interaction $V_{2}^{o}$ in the exchange channel. For a bounded density of states, and when the hybridization is large we expect the inequality $t>t_{p}$.

The scaling regime occurs within the energy range of $t_{p}$ near the Fermi level of the electron bath. At energy scales larger than this bandwidth $t_{p}$, we cannot apply the logarithmic RG based on decimation of the conduction electron bandwidth and perform instead a poor man's version of a realistic quantum chemical calculation. We first ignore $t_{p}$ and diagonalize the local problem and label the twelve eigenstates by the charge $N$ and the spin quantum numbers. The eigenvectors and the eigenvalues are:

$$
\begin{array}{cc}
\mid N=0> & \epsilon(N=0)=0 \\
\left|N=1, \sigma, b>=\left(u_{1} d_{\sigma}^{+}+v_{1} p_{\sigma}^{+}\right)\right| 0> & E(N=1, b)=\frac{1}{2}\left(\epsilon_{p}+\epsilon_{d}-R_{1}\right) \\
\left|N=1, \sigma, a b>=\left(-v_{1} d_{\sigma}^{+}+u_{1} p_{\sigma}^{+}\right)\right| 0> & E(N=1, a b)=\frac{1}{2}\left(\epsilon_{p}+\epsilon_{d}+R_{1}\right) \\
\left|N=2, s, b>=\left(u_{2} \epsilon_{\sigma \sigma^{\prime}} \frac{1}{\sqrt{2}} d_{\sigma}^{+} p_{\sigma^{\prime}}^{+}+v_{2} p_{\uparrow}^{+} p_{\downarrow}^{+}\right)\right| 0> & E(N=2, s, b)=\epsilon_{p}+\frac{1}{2}\left(\epsilon_{p}+\epsilon_{d}+V_{1}^{o}-V_{2}^{o}-R_{2}\right) \\
\left|N=2, s, a b>=\left(-v_{2}^{o} \epsilon_{\sigma \sigma^{\prime}} \frac{1}{\sqrt{2}} d_{\sigma}^{+} p_{\sigma^{\prime}}^{+}+u_{2} p_{\uparrow}^{+} p_{\downarrow}^{+}\right)\right| 0> & E(N=2, s, a b)=\epsilon_{p}+\frac{1}{2}\left(\epsilon_{p}+\epsilon_{d}+V_{1}^{o}-V_{2}^{o}+R_{2}\right) \\
\left|N=2, t>=d_{\sigma}^{+} p_{\sigma}^{+}\right| 0>, \frac{1}{\sqrt{2}} \sum_{\sigma} d_{\sigma}^{+} p_{-\sigma}^{+} \mid 0> & E\left(N=2, t>=\epsilon_{p}+\epsilon_{d}+V_{1}^{o}+V_{2}^{o}\right. \\
\left|N=3, \sigma>=d_{\sigma}^{+} p_{\uparrow}^{+} p_{\downarrow}^{+}\right| 0> & E(N=3)=\epsilon_{d}+2 \epsilon_{p}+2 V_{1}^{o}
\end{array}
$$


Here "b", "ab", "s" and "t" refer to bonding and antibonding combinations and singlet and triplet states respectively. The coherence factors are $u_{1}^{2}=1-v_{1}^{2}=\frac{1}{2}\left(1+\Delta / R_{1}\right)$ and $u_{2}^{2}=1-v_{2}^{2}=\frac{1}{2}\left(1+\left(\Delta-V_{1}^{o}+V_{2}^{o}\right) / R_{2}\right)$, where $\epsilon_{p}-\epsilon_{d}=\Delta$, $R_{1}=\sqrt{\Delta^{2}+4 t^{2}}$, and $R_{2}=\sqrt{\left(\Delta-V_{1}^{o}+V_{2}^{o}\right)^{2}+8 t^{2}}$.

As we vary the chemical potential, the energies of these local states vary. We consider the hole-doped regime, in which case $\epsilon_{p}$ is such that the local doublet $\mid N=1, \sigma, b>$ and the local Zhang-Rice-like singlet $\mid N=2, s, b>$ are almost degenerate, $E(N=1, b) \approx E(N=2, s, b)$. Such a mixed-valence condition determines $\epsilon_{p}$ to be $\epsilon_{p} \approx$ $\frac{1}{2}\left(R_{2}-R_{1}+V_{2}^{o}-V_{1}^{o}\right)$, which in turn specifies the energies for various excited states $\gamma: \epsilon(\gamma)=E(\gamma)-E(N=1, b)$.

They are given as follows,

$$
\begin{aligned}
& \epsilon(N=0)=\frac{1}{2}\left(2 R_{1}+\Delta-R_{2}+V_{1}^{o}-V_{2}^{o}\right) \\
& \epsilon(N=1, a b)=R_{1} \\
& \epsilon(N=2, s, a b)=R_{2} \\
& \epsilon(N=2, t)=\frac{1}{2}\left(R_{2}-\Delta+V_{1}^{o}+3 V_{2}^{o}\right) \\
& \epsilon(N=3)=\frac{1}{2}\left(2 R_{2}-\Delta-R_{1}+2 V_{1}^{o}+2 V_{2}^{o}\right)
\end{aligned}
$$

We now integrate out approximately the high energy eigenstates using second order perturbation theory to construct the low energy effective Hamiltonian:

$$
\begin{aligned}
\mathrm{H}_{\mathrm{eff}}= & \epsilon_{d}\left(\sum_{\sigma}|\sigma><\sigma|-|s><s|\right)+\tilde{t}\left(\sum_{\sigma}|\sigma><s| p_{-\sigma}^{+} \operatorname{sgn}(\sigma)+\text { h.c. }\right) \\
& -\tilde{V}_{1} \sum_{\sigma, \sigma^{\prime}}|\sigma><\sigma| p_{\sigma^{\prime}}^{+} p_{\sigma^{\prime}}+\frac{\tilde{V}_{2}}{4} \sum_{\sigma_{1}, \sigma_{2}, \sigma_{3}, \sigma_{4}} \tau_{\sigma_{1} \sigma_{2}} \cdot \tau_{\sigma_{3} \sigma_{4}}\left|\sigma_{1}><\sigma_{2}\right| p_{\sigma_{3}}^{+} p_{\sigma_{4}}
\end{aligned}
$$

with the various parameters given by:

$$
\begin{aligned}
& \tilde{V}_{1}=\frac{1}{2}\left(t_{01}^{p}\right)^{2}\left(-\frac{v_{1}^{2}}{\epsilon(N=0)}+\frac{3}{2} \frac{u_{1}^{2}}{\epsilon(N=2, t)}-\frac{u_{2}^{2}}{\epsilon(N=3)}+\frac{2}{\epsilon(N=1, a b)}\left(v_{2} u_{1}-\frac{v_{1} u_{2}}{\sqrt{2}}\right)^{2}\right) \\
& \tilde{V}_{2}=\frac{1}{2}\left(t_{01}^{p}\right)^{2}\left(\frac{v_{1}^{2}}{\epsilon(N=0)}-\frac{1}{2} \frac{u_{1}^{2}}{\epsilon(N=2, t)}+\frac{1}{\epsilon(N=2, s, a b)}\left(v_{1} u_{2}-\frac{u_{1} v_{2}}{\sqrt{2}}\right)^{2}\right) \\
& \tilde{t}=v_{1} t
\end{aligned}
$$

By using a suggestive notation, we define a new vacuum $|v a c\rangle=s^{+} \mid 0>$, and creation operators for the doublet $d_{\sigma}^{+}=|\sigma><s|$ which obey a no double occupancy constraint. After performing a particle-hole transformation $p_{-\sigma}^{+} \operatorname{sgn}(\sigma) \rightarrow p_{\sigma}$, the effective Hamiltonian given in Eq. (12) reduces to the Hamiltonian, Eq. (3) described in the previous section with $V_{1}=\tilde{V}_{1}$ and $V_{2}=\tilde{V}_{2}$. We can now apply the RG analysis to determine the basin of attraction of the various phases. As stated above it is natural to express the answers in terms of the effective phase shifts $\tilde{\delta}_{1}=\tan ^{-1}\left(\pi \rho \tilde{V}_{1}\right)$ and $\tilde{\delta}_{2}=\tan ^{-1}\left(\pi \rho \tilde{V}_{2}\right)$. The system renormalizes into the weak coupling non-Fermi liquid state if $\tilde{\delta_{1}} / \pi<-(\sqrt{3}-1) / 2$ and $\tilde{\delta_{2}}<0$, and into the intermediate coupling non-Fermi liquid state if $\tilde{\delta_{1}} / \pi<-(\sqrt{3}-1) / 2$ and $\tilde{\delta_{2}}>0$.

The effective interactions, given in Eq. (13), depend on the way high energy states arrange among themselves which in turn is determined by the quantum chemistry (i.e. the initial parameters in the full impurity model, Eq. (9)) of 
the problem. The basin of attraction of the non-Fermi liquid states occurs when $\epsilon(N=0)$ or $\epsilon(N=3)$ is small. It is easy to see from Eq. (9) that, $\epsilon(N=0)$ is small for a strong antiferromagnetic $V_{2}^{o}$ and a weak repulsive $V_{1}^{o}$. We explored the parameter space $t, V_{1}^{o}$ and $V_{2}^{o}$ for a given $\Delta$, and found that for large $V_{2}^{o}$ and small $V_{1}^{o}$ the non-Fermi liquid regime is larger when the hybridization increases.

In Fig. 11, we plot $\tilde{V}_{1} /\left(t_{01}\right)^{2}$ and $\tilde{V}_{2} /\left(t_{01}\right)^{2}$ as a function of $V_{2}^{0}$ for a $\Delta=2, t=1$, and $V_{1}^{0}=0$. It can be seen that, $\tilde{V}_{1} /\left(t_{01}\right)^{2}$ turns to negative when $V_{2}^{0}$ is large enough, and diverges upon approaching $\left(V_{2}^{0}\right)_{c} \approx 2.415$ at which $\epsilon(N=0)=0$. This divergence is not physical but indicates the breakdown of second order perturbation theory. Qualitatively we can say that the intermediate phase is realized when the quantum chemistry of the problem is such as to have a low lying excited "empty sate". This happens when the Kondo exchange which stabilizes the Zhang-Rice singlet is large and when the hybridization $t$ is comparable to $\Delta$.

Finally, we observe that, in finite dimensions, the incoherent metallic states cannot be stable at zero temperature. The algebraic decay of the local correlation functions are symptomatic of a superconducting instability that sets in at a finite energy scale $T_{c}$. The feedback of the long range order over the single particle properties is down by powers of $\frac{1}{d}$. In finite dimensions the coupling of the long range order on the local physics modifies the local effective action at temperatures $T<T_{c}$, and has the effect of cutting off the power law singularities. Nevertheless, the incoherent metallic states have a well defined existence at temperatures $T_{c}<<T<<\omega_{c}$ in the same sense that the paramagnetic Mott insulating phase is well defined only for $T_{N}<<T<<\epsilon_{F}, T_{N}$ being the Neel temperature.

The ideas presented here approach the problem of the breakdown of Fermi liquid in strongly correlated fermion systems in the same spirit that the combination of the Anderson-Yuval RG calculation coupled to the Nozieres strong coupling fixed point analysis solves the antiferromagnetic Kondo problem. It provides a conceptual framework, in which one solves a complicated problem by treating it with different tools at different energy scales. The Kondo problem involves the spontaneous generation of a scale. Therefore it is self-similar at short distances, and requires strong coupling methods at long distances. Here we find ourselves in the opposite situation: the problem is to understand the generation of a scale invariant self-similar low energy regime. The strong coupling methods are applied at high energy scales, and the renormalization group, when started with the correct quantum chemistry initial conditions, drives us towards the weak coupling self-similar regimes.

ACKNOWLEDGMents: We thank J. Cardy, A. Finkelstein, A. Georges, T. Giamarchi, A. Ruckenstein, and C. Varma for useful discussions. This work was supported by the NSF under grant DMR 922-4000.

$1 \quad$ P. W. Anderson, Science 235, 1196 (1987); Physica C185-189, 11 (1991).

2 A. E. Ruckenstein and C. M. Varma, Physica C185-189, 134 (1991).

3 J. Solyom, Adv. Phys. 28, 201 (1979); V. J. Emery, in Highly Conducting One-dimensional Solids, Eds. J. T. Devreese et al. (Plenum, New York, 1979); F.D.M. Haldane, J. Phys. C14, 2585 (1981). 
R. Shankar, preprint (1992).

5 J. Hubbard, Proc. Roy. Soc. A281, 401 (1964).

6 For recent reviews, see D. Vollhardt, Jerusalem Winter School lecture notes (1992); A. Georges, G. Kotliar and Q. Si, Int. Journ. Mod. Phys. B6, 705 (1992) and references therein.

$7 \quad$ A. Georges and G. Kotliar, Phys. Rev. B45, 6479 (1992).

8 M. J. Rozenberg, X.Y. Zhang, and G. Kotliar, Phys. Rev. Lett. 69, 1236 (1992); A. Georges and W. Krauth, ibid., 1240 (1992); M. Jarrell, ibid., 168 (1992).

9 Q. Si and G. Kotliar, Phys. Rev. Lett. 70, xxxx (1993); preprint (1993).

10 F.D.M. Haldane, Phys. Rev. Lett. 40,416(1978) and Ph.D Thesis, Univ. of Cambridge, 1977 (unpublished); J Phys. C11, 5015 (1978).

11 P.W. Anderson and Z. Zou, Phys. Rev. Lett. 60, 132 (1988); L. B. Ioffe and A. Larkin, Phys. Rev. B39, 8988 (1989); N. Nagaosa and P. A. Lee, Phys. Rev. Lett.64, 2450(1990); L. B. Ioffe and G. Kotliar, Phys. Rev. B42, 10348 (1990).

12 For a review, see A. J. Leggett et al., Rev. Mod. Phys. 59, 1 (1987).

13 Q. Si, G. Kotliar, and A. Georges, Phys. Rev. B46, 1261 (1992).

14 Q. Si and G. Kotliar, (unpublished).

15 J. L. Cardy, J. Phys. A14, 1407 (1981). See also S. Chakravarty and J. Hirsch, Phys. Rev. B 25, 3273 (1982).

16 The scaling equations show that, it is impossible to have spin kinks bound while charge kinks are unbound.

17 D. Nelson, in Phase Transitions and Critical Phenomena, Vol. 7, p. 1, eds. C. Domb and J.L. Lebowitz (Academic Press, Londo, 1983).

18 T. Giamarchi, C. Varma, A. Ruckenstein and P. Nozieres, preprint (1992); I. Perakis, C. Varma, and A. E. Ruckenstein, preprint (1993). 
FIG. 1. Effective interactions $\tilde{V}_{1} /\left(t_{01}^{p}\right)^{2}$ (solid line) and $\tilde{V}_{2} /\left(t_{01}^{p}\right)^{2}$ (dashed line) as a function of the "bare" interaction $V_{2}^{0}$ defined in the text. The parameters are $\Delta=2, t=1$, and $V_{1}^{0}=0$. 